\title{
Influence of interionic separation in electron transfer reactions
}

\author{
Alfredo P. Carvalho, M. Natália D.S. Cordeiro*, José A.N.F. Gomes \\ CEQUP/Departamento Química, Faculdade de Ciências do Porto, Rua do Campo Alegre 687, 4169-007 Porto, Portugal
}

Received 26 September 1998; received in revised form 7 December 1998; accepted 8 December 1998

\begin{abstract}
A theoretical study is presented on the influence of the interionic separation between the reacting metal cations on the kinetics of electron transfer reactions. The results of some simulations are presented to characterize the dependence of the activation free energy of the electron transfer process relatively to the interionic separation as well as the potential of mean force between the two cations considered in this study, $\mathrm{Cu}^{+}$and $\mathrm{Cu}^{2+}$, in aqueous medium. The use of truncation of coulombic interactions is compared with the use of Ewald sums in the treatment of long range interactions. The truncation of coulombic interactions in the simulations is observed to have a strong influence in the final results. (C) 1999 Elsevier Science B.V. All rights reserved.
\end{abstract}

Keywords: Pmf; Electron transfer; Copper; Ionic solutions; Monte Carlo

\section{Introduction}

The study of ionic interactions in aqueous medium is fundamental for the understanding of many chemical phenomena. The solvent mediation of the interaction between reactant ionic species usually has a determining role in the outcome of chemical processes.

Electron transfer reactions between metal cations in aqueous medium is an example of one such kind of processes that are mainly driven by fluctuations of the solvent configurations. In particular, the solvent's intervention is of crucial importance in the necessary approach of the reactant cations and, afterwards, in the electron transfer process itself as it is understood within the Marcus' theoretical framework [1-4].

The influence of the interionic distance in the free energy of activation of the electron transfer process has usually been neglected and not studied in detail.

* Corresponding author.

E-mail address: ncordeir@fc.up.pt (M. Natália D.S. Cordeiro)
The electron hopping frequency is naturally favored by small separations between the metallic centers. One can also expect the most determining step - the solvent reorganization - to be somewhat favored when the cations approach, as each cation, when at close separation, may perturb the other cation's hydration shell in a way which makes it easier for the solvent to reorganize for the electron transfer.

The most convenient way to characterize the energetic profile for the cation's approach, that will determine the reaction's interionic separation, is the calculation of a potential of mean force (PMF) of the two solvated metal ions.

The in vacuum repulsive electrostatic interaction between like-signed ions or charged sites in molecules may, in solution, be balanced with solvation interactions, thus allowing such charged centers to be, in average, closer to each other [5-10]. Early works on the theoretical description of ionic interactions in solution have yielded PMFs revealing a short range attractive behavior between like-signed ions [8-11], in particular between anions, which was explained by 
Table 1

Parameters for the ion-water dispersion terms of the potential functions. Values given in atomic units

\begin{tabular}{lclcl}
\hline Parameters & $\mathrm{Cu}^{2+}-\mathrm{O}$ & $\mathrm{Cu}^{2+}-\mathrm{H}$ & $\mathrm{Cu}^{+}-\mathrm{O}$ & $\mathrm{Cu}^{+}-\mathrm{H}$ \\
\hline$A$ & 79.63 & 0 & 0.6990 & -0.3495 \\
$B$ & 1.919 & 0 & -17.03 & 8.513 \\
$C$ & 27.49 & 0 & 205.6 & -18.57 \\
$D$ & -104.25 & 0 & -1078 & 114.2 \\
$E$ & 1441 & 0 & 1940 & -171.9 \\
$F$ & - & - & -71281 & 1832 \\
\hline
\end{tabular}

a bridging effect established by the solvation shells' water molecules. Experimental studies, however, contradicted these theoretical findings. The analysis of distinct diffusion coefficients [12] as well as data concerning electrolyte mixing thermodynamics [13] for the chloride ion pair case, did not support the ion pairings. As noted by some researchers [14,15], for high dielectric solvents the solvent contribution to the PMF is nearly identical, in magnitude but opposite in sign to the ionic interaction in vacuum, which makes PMFs a delicate and very sensitive balance between similar but opposing interactions. It happens that in earlier simulations, the long range electrostatic interactions were commonly truncated for distances longer than half the simulation box length. Following works, where an appropriate treatment of the long range interactions was used, either by Ewald sums or reaction field techniques [16-18], all yielded essentially repulsive PMFs or with very shallow minima, therefore providing much weaker support for the existence of those ion pairs. It has been observed $[15,19]$ that results obtained by use of truncated electrostatics can be in significant disagreement with those obtained using Ewald techniques.

The use of Ewald sums in simulations of electrically charged systems usually raises important objections, as the sums can only be meaningfully applied to neutral systems. However, the usual formulation of the Ewald sums includes a term corresponding to a background with a charge opposite to the system's charge, thus making the overall system neutral $[20,21]$. In these conditions, Ewald sums are, therefore, appropriate for the treatment of systems even if they are electrically charged.

This work presents the PMF of $\mathrm{Cu}^{+}-\mathrm{Cu}^{2+}$ in aqueous solution calculated by Monte Carlo simulations. A brief comparison is made between results obtained by truncation of electrostatic interactions and their treatment by the Adams and Dubey's approximation to the Ewald sums [22]. An attempt is made to clarify whether the appropriate treatment of long range interactions affects PMFs so dramatically simply due to the result of the addition of a greater number of long range ion-water interactions or if there is any important structural changes in the hydration shells of the ions owing to long range correlations.

Results are also presented of simulations of the electron transfer process $\mathrm{Cu}^{2+}+\mathrm{Cu}^{+} \rightleftharpoons \mathrm{Cu}^{+}+$ $\mathrm{Cu}^{2+}$ in aqueous solution at several different interionic separations of the pair $\mathrm{Cu}^{+} / \mathrm{Cu}^{2+}$ in order to characterize the influence of this parameter in the kinetics of this process. The results of these simulations are discussed in complement with the PMF calculation.

In Section 2, the model used in the calculations is described. The PMFs are presented in Section 3.1 and an analysis is carried out of the structure of the hydration shells of the ions at several key separations. The results of the electron transfer simulations at several interionic separations are presented in Section 3.2. These results are discussed in Section 4 along with the presentation of some concluding remarks.

\section{Computational models}

The system under study is composed of a $\mathrm{Cu}^{+}$and a $\mathrm{Cu}^{2+}$ cations solvated by 200 water molecules in the NVT ensemble at $298 \mathrm{~K}$.

The interactions between the particles of the system were described by pairwise additive potential functions, thus neglecting higher order terms for the potential functions such as three-body terms.

The water-water interactions were described by the SPC water model [23] which has been widely used and proven to give good results in condensed phases.

The $\mathrm{Cu}^{2+}$ cation-water interactions were described by the potential function proposed by Curtiss et al. with dispersion terms given by the expression [24]

$V_{i j}^{\mathrm{disp}}=A_{i j} e^{-B_{i j} r i j}-\frac{C_{i j}}{r_{i j}^{4}}-\frac{D_{i j}}{r_{i j}^{6}}-\frac{E_{i j}}{r_{i j}^{12}}$

with the values of the parameters $A-E$ given in Table 1. This potential function has been tested by Cordeiro 
[25] producing a coordination number of $6[26,27]$ but an overestimated solvation energy in comparison with the available experimental data [28].

The $\mathrm{Cu}^{+}$cation-water interactions were described by the potential function suggested by Cordeiro et al. [29] with dispersion terms given by the expression

$$
V_{i j}^{\mathrm{disp}}=\frac{A_{i j}}{r_{i j}^{2}}+\frac{B_{i j}}{r_{i j}^{3}}+\frac{C_{i j}}{r_{i j}^{4}}+\frac{D_{i j}}{r_{i j}^{5}}+\frac{E_{i j}}{r_{i j}^{6}}+\frac{F_{i j}}{r_{i j}^{12}} .
$$

with the values of the parameters $A-F$ given in Table 1.

In the original potential function of Cordeiro and co-workers, as presented in Ref. [29], a $r^{-1}$ term of the polynomial was also parameterized in the fitting to the quantum energy curve. The parameters presented in Table 1 are slightly modified relatively to the original values so that the parameters of the $r^{-1}$ terms can be held fixed as the products $q_{O} q_{C u^{+}}$and $q_{H} q_{C u^{+}}$, allowing for a purely coulombic sense to be attributed to that term and a consistent and meaningful treatment of the electrostatic interactions to be carried out. However, this potential yields similar results to the original one [29].

The $\mathrm{Cu}^{2+}-\mathrm{Cu}^{+}$interaction for the PMF calculation was described just by an electrostatic term. Notice that this interaction is irrelevant for the electron transfer simulations. Considering that the two particles constitute a highly charged couple, the electrostatic interactions should be of a much larger magnitude than the dispersion terms for all but very short separations. Therefore the neglect of such terms should only cause a significant underestimation of the repulsion between those two ions at very close distances which are of little importance for this kind of study. In any case, other cation-cation interaction terms can always be added to the PMF a posteriori.

The long range interactions were calculated for the case of an infinite size system composed of replicas of the simulation box, following an Ewald sum scheme. However, instead of the usual methods for the calculation of the Ewald energy, an approximation was used employing Kubic Harmonics functionals which were adjusted to the exact Ewald values by Adams and Dubey [22]. This approximation allows much faster calculations to be performed in comparison to the ordinary methods of calculating the Ewald values [30]. The level of accuracy of the approximation can be chosen at the expense of computational speed, allowing for a tradeoff to be made between accuracy and speed. This approximation has been successfully tested in simulations of molten salts [31], one component plasma [22] and polar liquids [32-34]. Therefore, it seems to be a suitable way to account for long range electrostatic interactions in an almost Ewald manner.

In the present work, the length of the Kubic Harmonics expressions, which controls the speed of calculation as well as the level of accuracy, was taken up to the 10th power, which produces RMS errors relatively to the true Ewald values of about $6.2 \times 10^{-4}$ [22].

From now on in this article the results obtained using this Adams and Dubey's method will be referred to as Ewald results although they really correspond to this approximation to the true Ewald expression.

These long range interactions were, however, only calculated for the ion-water and water-water electrostatic interactions. As the system under study is supposed to simulate an aqueous solution of $\mathrm{Cu}^{+}$ and $\mathrm{Cu}^{2+}$ at infinite dilution, the interaction between those two ions in the PMF is taken as a single coulombic interaction, as the interactions with the images are meaningless. In addition, considering the infinite dilution of the system, the possibly relevant inclusion of counter-ions should not introduce any desirable influence in this simple model system.

There has been some debate in the past over the importance of the type of boundary surrounding the system. It has been argued in a couple of interesting articles [21,35] that, for different types of properties studied, the influence of the surrounding medium considered has varying influence. These range from the nearly negligible dependence on the boundaries to the most pronounced sensitivity to the type of surrounding medium used (whether vacuum or tinfoil). Some properties seem to be greatly dependent on the effect of replication of the simulation box whereas others seem to be more influenced by the boundary. In the last case, the use of tinfoil boundaries seem to be more appropriate for the correct calculation of certain properties whereas vacuum boundaries seem to be preferable in other situations [35]. In the present work, the tinfoil boundary was considered to be the best choice, but a more careful study on the effect of the boundary could be appropriate. 
In addition, a second PMF was calculated where, instead of using Adams and Dubey's scheme, the electrostatic interactions were simply truncated for distances bigger than half the length of the simulation box and periodic boundary conditions were used, so that results obtained by both methods could be compared. The results obtained in this way will be referred to as truncated electrostatics (TE) results. The PMFs were calculated by a series of Metropolis Monte Carlo simulations [36] using a Free Energy Perturbation methodology [37].

For the calculation of each PMF, 35 simulations were computed, where the cations were sequentially moved apart (or towards each other) by steps of $0.1 \AA$ from a separation of $3.0 \AA$ up to $6.5 \AA$. Independent calculations of the PMFs were carried out for both the separating and the approaching directions. As doublewide sampling [38] was not used, the present PMFs are the result of averaging both PMFs. As pointed out by Clementi [39], averaging independent forward and backward runs results in statistically better PMFs. In the beginning of each PMF, an extra simulation was performed with the generation of $16 \times 10^{6}$ configurations, to allow for an initial equilibration of the system.

As a result of the volume of computation involved in this work, the system has a small size. Therefore, the PMF cannot be calculated to an extension much bigger than $7 \AA$. However, our main interest is the short range features of the PMF corresponding to the first solvation shell and some tests made with larger systems (up to $\approx 400$ water molecules) have not revealed significant changes.

For each simulation, the last configuration of the preceding simulation was used as its starting configuration, and $4 \times 10^{6}$ configurations were generated for equilibration of the system. Properties were then averaged over additional $12 \times 10^{6}$ configurations.

The simulation box for the TE case was a rectangular box with dimensions of $17 \AA \times 17 \AA \times 22 \AA$ and with the longer axis taken as the interionic separation axis. For the Ewald case, the box used was a cube with sides of $18 \AA$. The use of a cube in the latter case was imposed by the nature of the Kubic Harmonics functionals used in the Adams and Dubey's approximation to the Ewald sums [22].

The electron transfer simulations were conducted using of a special mapping potential of the form

$U_{m}=\left(1-\lambda_{m}\right) U_{r}+\lambda_{m} U_{p}$

which is used as the effective ion-water potential instead of the ordinary potential used to calculate the PMF. $\lambda$ is slowly varied from 0 to 1 in a way that converts a reference system (described by the potential $U_{r}$ ) when $\lambda=0$ into a perturbed system (described by the potential $U_{p}$ ) when $\lambda=1$. In the present case, the reference system corresponds to a initial state composed by the reactants and the perturbed system corresponds to a final state composed by the products. It should be noted, however, that as the reaction is symmetric, $\lambda$ needs only to be varied from 0 to 0.5 .

The parameter $\lambda$ has been incremented throughout the process according to the formula [39]

$$
\begin{aligned}
\lambda_{m+1} & =\frac{1}{2}(1+\tanh ((-N / 2+m) W)), \\
m & =0, N-1
\end{aligned}
$$

where $N$ is the total number of steps. The constant $W$ was given the value of 0.02 which ensures that the free energy changes are very small in the initial and final steps.

The free energies $\Delta A\left(\lambda_{r} \rightarrow \lambda_{m}\right)$ (or $\Delta A\left(\lambda_{p} \rightarrow \lambda_{m}\right)$ ) were calculated by the common thermodynamic perturbation method. A more detailed description of the method can be consulted in [40].

Two simulations were conducted, where the two ions are separated by 5 and $7 \AA$. The two ions were placed at the center of a cube with a side of $18 \AA$. Each of the two simulations consisted of $20 \lambda$ steps of $24 \times 10^{6}$ configurations each, half being used to equilibrate the system and the other half to produce the averages. For the case of the electron transfer at $7 \AA$, a calculation has already been done in a previous work [40] using a truncation of the electrostatic interactions which now can be compared with the present results with a different treatment of the long range interactions.

The averages in the simulations were calculated by the block means method as proposed by Bishop and Frinks [41]. The statistical errors in the calculations were assumed to have a normal distribution in the simulations. These errors were estimated 


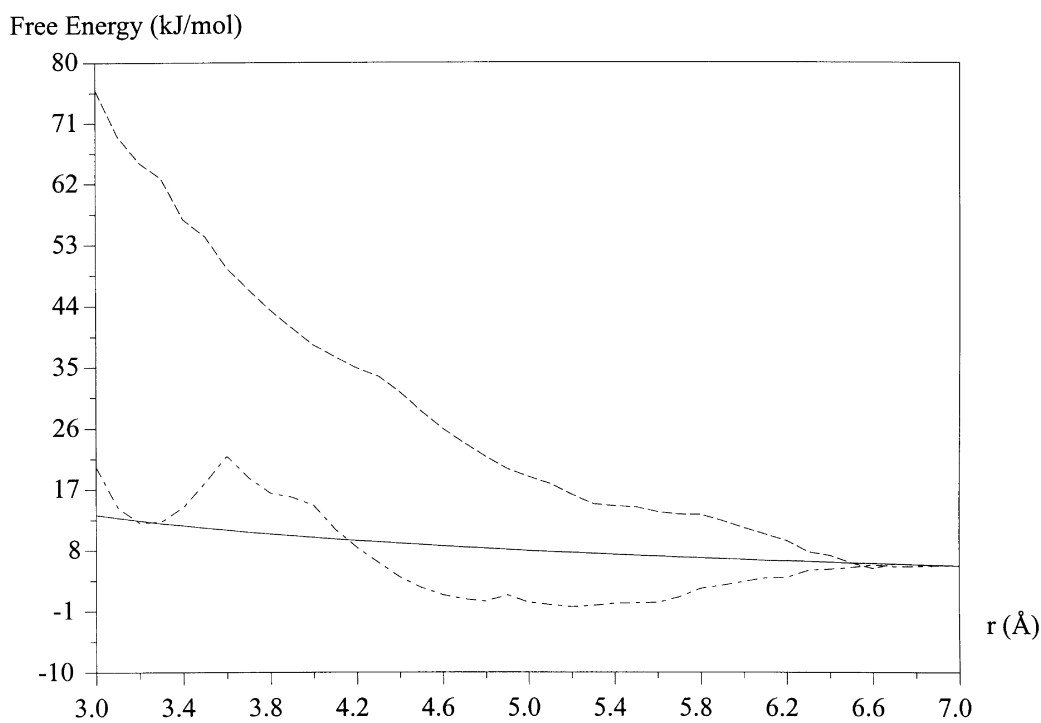

Fig. 1. $\mathrm{Cu}^{+}-\mathrm{Cu}^{2+} \mathrm{PMF}$ calculated with use of Ewald sums (dashed line) and with the truncation of the electrostatic interactions (dot-anddashed line). In solid line the dielectric continuum case is depicted using the SPC dielectric constant $\epsilon=70$.

according to the Straatsma and McCammon's method [42].

\section{Results}

\subsection{Potential of mean force}

Fig. 1 presents the results of the PMF calculation carried out using the truncated electrostatic interactions. It is also shown, for comparison, the PMF of the ions in a continuum dielectric as given by $q_{1} q_{2} / \epsilon \mathrm{r}_{12}$ with $\epsilon=70$ for the static dielectric constant of the SPC water [43]. As this last PMF is the asymptotic limit for long separations, the calculated PMF was anchored to the continuum values at the longest distance of $7 \AA$. As can be seen, two local minima are produced in the free energy curve at $3.2 \AA$ and $5.2 \AA$. While the first minimum, at contact distance, is narrow and above the continuum dielectric case, the second minimum is very broad and goes below it. The solvent separated minimum is $12.5 \mathrm{~kJ} / \mathrm{mol}$ below the contact minimum and a barrier of $22.3 \mathrm{~kJ} / \mathrm{mol}$ separates the minimum at $5.2 \AA$ from the contact minimum at $3.2 \AA$. One point to note is that this PMF, at large separations, seems to tend to the correct asymptotic behavior, unlike other results for analogous systems such as those obtained by Bader and Chandler for the $\mathrm{Fe}^{2.5+}-\mathrm{Fe}^{2.5+}$ system [15].

Also in Fig. 1 are displayed the results of the PMF calculation with the Adams and Dubey's treatment of long range interactions. By taking into account long range interactions in this manner, it can be observed that the profile of the PMF is drastically changed. No minima are observed in the PMF, neither at contact nor at solvent separated distances, and it smoothly approaches the continuum limit for distances bigger than $5 \AA$. Thus, the PMF always remains repulsive if long range interactions are treated by Ewald sums, in agreement with the Bader and Chandler's findings [15].

In general, the structure of the solvent shell of $\mathrm{Cu}^{+}$ when truncation of long range interactions is applied suffers a smaller deformation as result of the influence of its $\mathrm{Cu}^{2+}$ neighbor in comparison with the solvation shell under Ewald. This effect can be clearly noted by comparing the average orientations of the solvent molecules which form the hydration shell of each cation. This orientation can be determined from the inner product between the position vector relatively to the cation $(\vec{r})$ and the water $\mathrm{C}_{2} v$ axis unitary vector $(\vec{f})$,

$O P=\left\langle\frac{\vec{r} \cdot \vec{f}}{|\vec{r}|}\right\rangle$, 


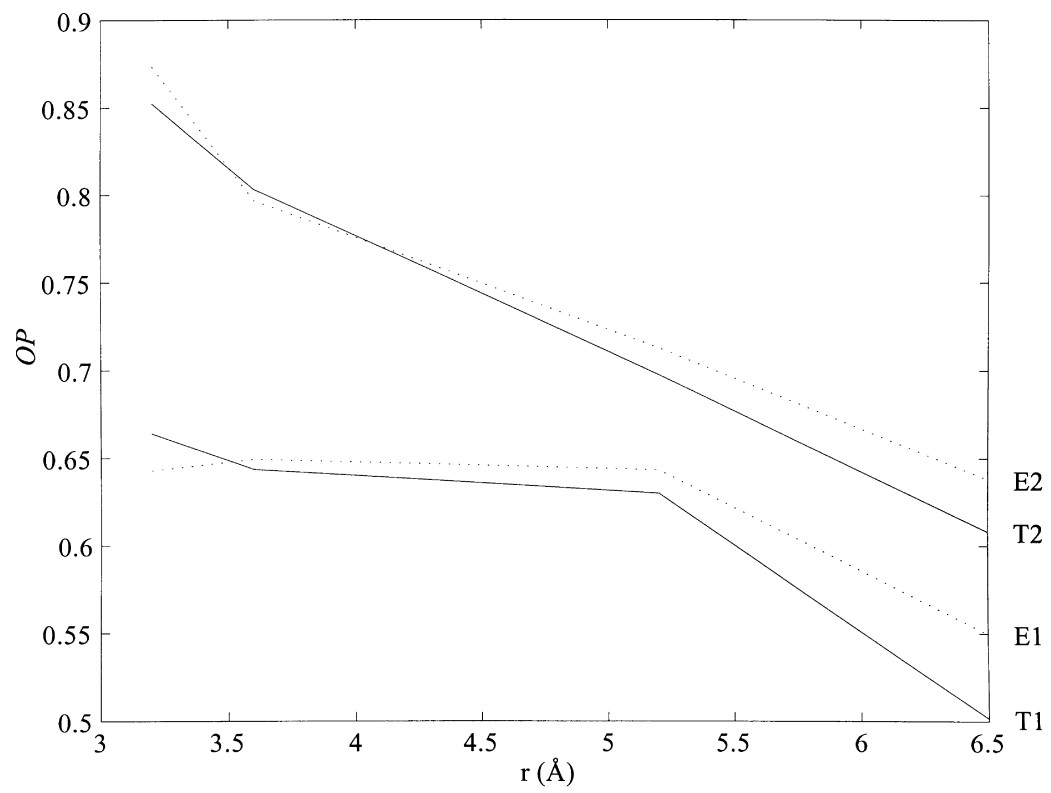

Fig. 2. Orientation parameters (Eq. (5)) of the hydration shell waters of $\mathrm{Cu}^{+}$and $\mathrm{Cu}^{2+}$ resulting from TE (solid line) and Ewald (dotted line)

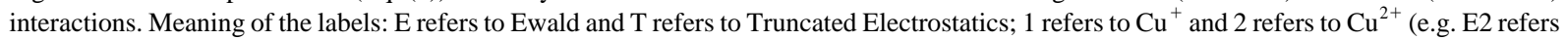
to $\mathrm{Cu}^{2+}$ using Ewald).
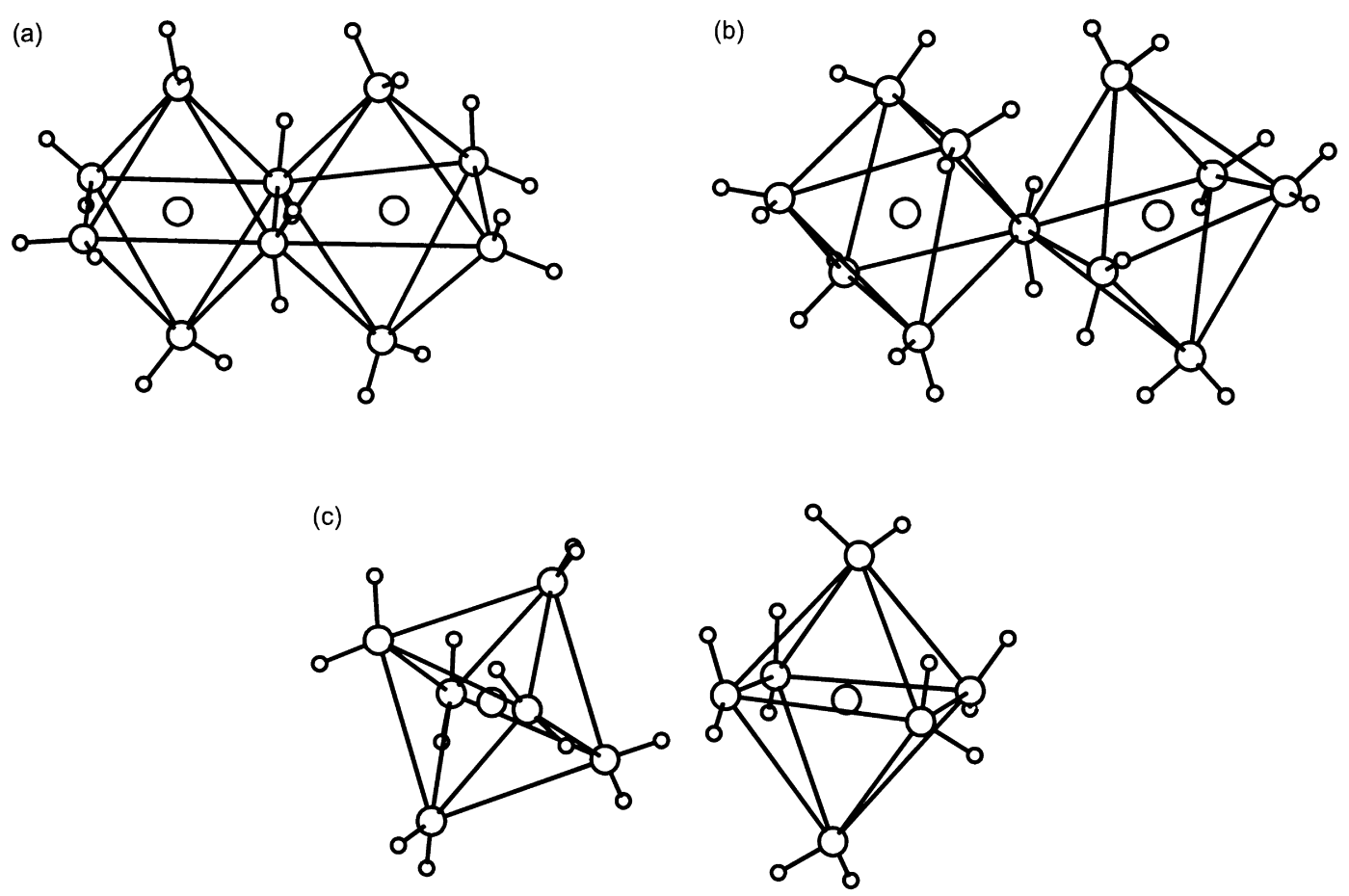

Fig. 3. Sample snapshots of the hydration shells at interionic separations of (a) $3.2 \AA$, (b) $3.6 \AA$ and (c) $5.2 \AA$. These configurations were obtained with Ewald sums and intend to illustrate the water molecules shared at the shorter interionic separations. 


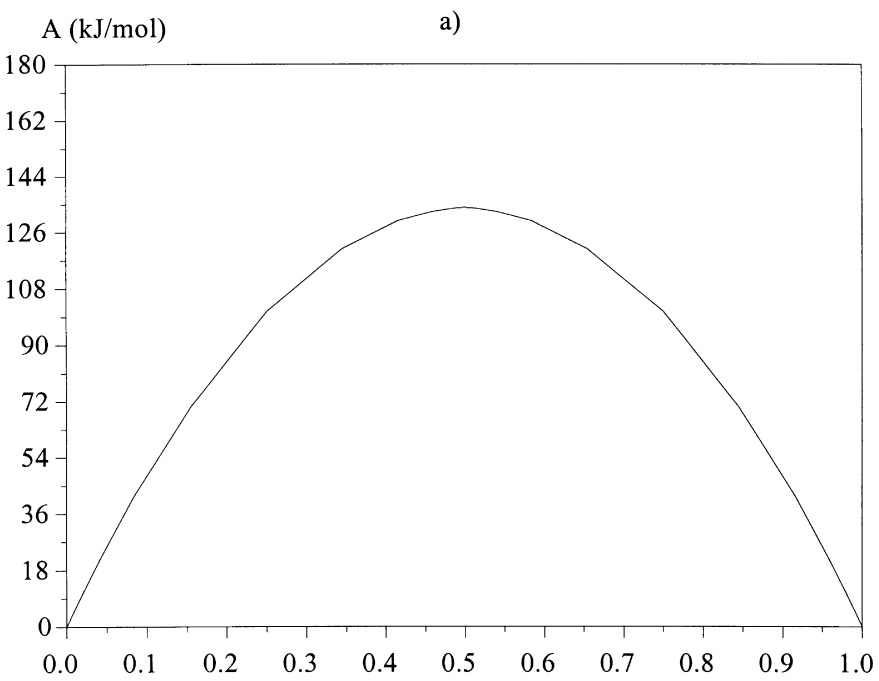

lambda

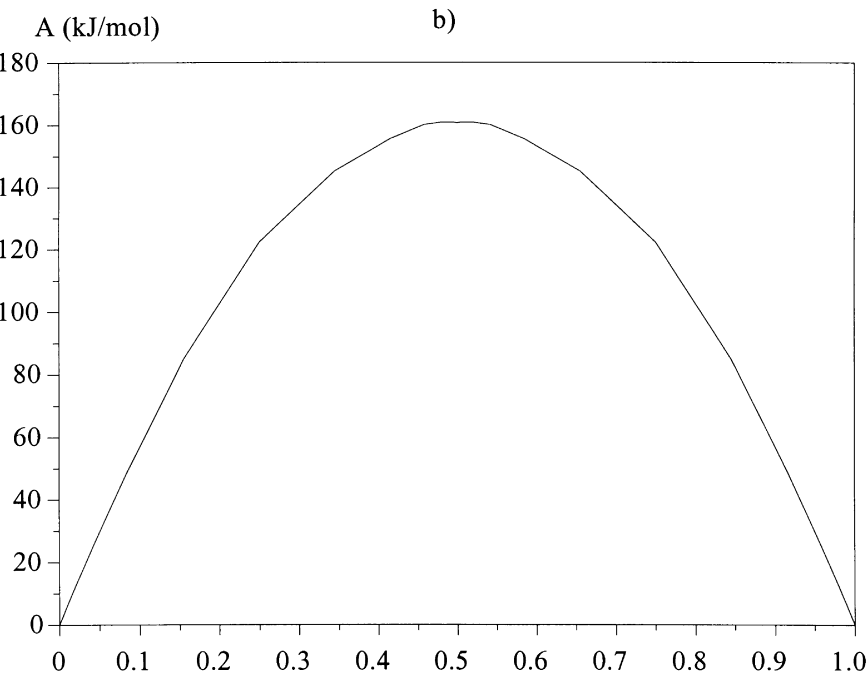

lambda

Fig. 4. Free energy as a function of the parameter $\lambda$ which is varied in the simulations. (a) Results for a interionic separation of $5 \AA$. (b) Results for a interionic separation of $7 \AA$.

yielding an orientation parameter $O P$ which is the cosine of the orientation angle of the water molecules. The value of $O P$ will be 1 when the water oxygens are oriented towards the cation and the hydrogen are oriented outwards, -1 when it is the opposite way and 0 when the $\mathrm{C}_{2} v$ axis is perpendicular to the position vector.

From the observation of Fig. 2 it can be seen that, as expected, the $\mathrm{Cu}^{2+}$ cation has a strongly perturbative influence on the $\mathrm{Cu}^{+}$hydration shell so that the water molecules always have a much weaker orientation towards $\mathrm{Cu}^{+}$so as to gain a more favorable orientation towards $\mathrm{Cu}^{2+}$.

When the ions move apart from each other, the water molecules in the interionic region lose orientation as the two hydration shells cease to share water molecules and the two independent shells begin to superimpose on each other. From the comparison of the TE case with the Ewald case it can be observed that the most striking changes occur at the largest 


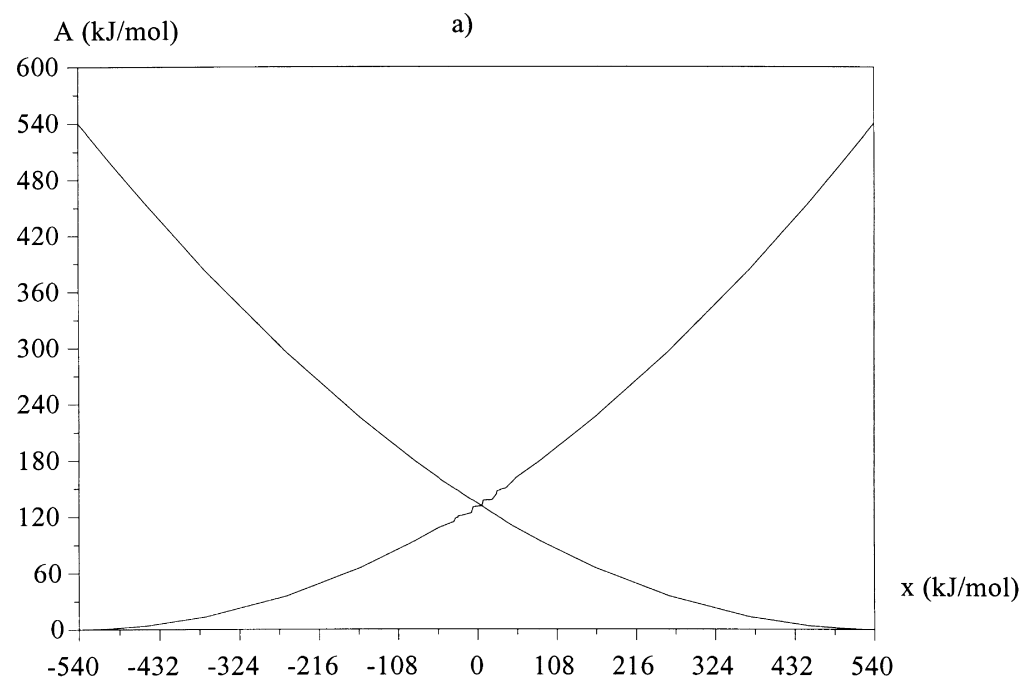
A $(\mathrm{kJ} / \mathrm{mol})$

b)

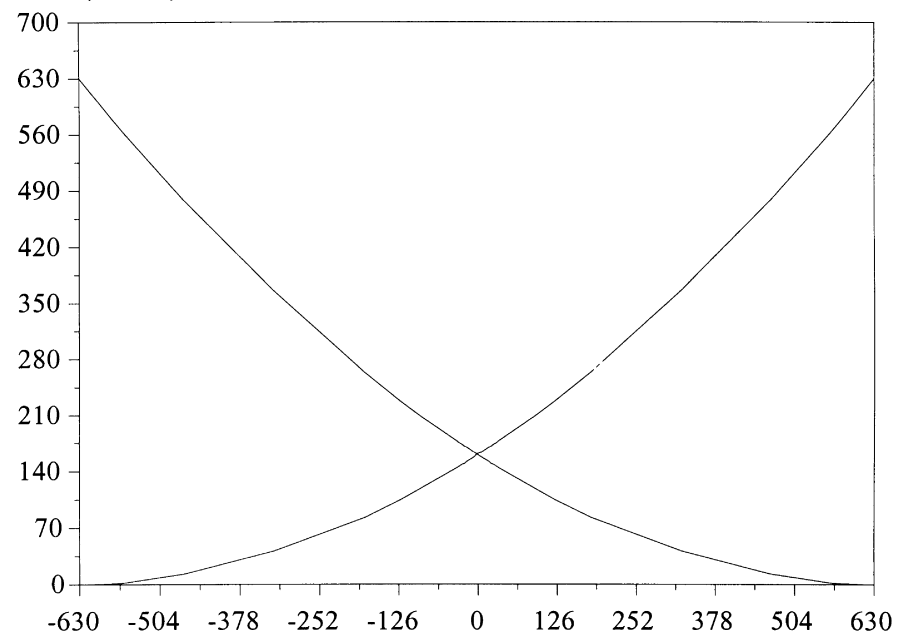

$\mathrm{x}(\mathrm{kJ} / \mathrm{mol})$

Fig. 5. Diabatic free energy curves for reactants and products as functions of the reaction coordinate $x=U_{p}-U_{r}$. (a) Results for a interionic separation of $5 \AA$. (b) Results for a interionic separation of $7 \AA$.

separations -5.2 and $6.5 \AA$. The two hydration shells share two water molecules at $3.2 \AA$, share one molecule at $3.6 \AA$ and are independent at ca. $5 \AA$ (see Fig. 3 ). As this process proceeds, the waters between the cations have to twist their orientations to lower the hydrogen-hydrogen repulsions between the two shells. This effect is more pronounced for separations beyond $5 \AA$ for the $\mathrm{Cu}^{+}$shell. The influence of the truncation of electrostatic interactions makes itself noticeable in a clearly poorer orientation of the shells which leads to weaker cation-water interactions. It can also be seen that from $3.2 \AA$ to $3.6 \AA$ the waters around the $\mathrm{Cu}^{+}$cation keep their orientations under Ewald whereas under TE their orientations follow the diminishing trend.

\subsection{Electron transfer}

In Fig. 4, the free energies $\Delta A_{r} \rightarrow{ }_{m}$, calculated by the thermodynamic perturbation method, are 
presented. From the values reported in Fig. 4, the diabatic free energy curves of the reactants and products, $\Delta A_{r}(x)$ and $\Delta A_{p}(x)$ respectively, were computed $(x$ being the reaction coordinate, which corresponds to the difference between the potential energies of the product and reactant states, $x=U_{p}-$ $U_{r}$ ) and are shown in Fig. 5. The diabatic free energy curves were computed according to the scheme used by Warshel and co-workers [44] (see also [40]).

Both free energy curves in Fig. 5 appear to have the parabolic behavior expected from the Marcus theory. The activation free energies $\Delta A^{\neq}$for the two cases are $133.9 \pm 7.3 \mathrm{~kJ} / \mathrm{mol}$ for the $5 \AA$ separation system and $160.8 \pm 9.2 \mathrm{~kJ} / \mathrm{mol}$ for the $7 \AA$ one. The latter value is substantially lower than the one obtained for the same separation in a previous study where truncation of long range interactions were used $(196.9 \mathrm{~kJ} / \mathrm{mol}$ [40]). In the present study, the electron transfer process occurs more favorably at $5 \AA$ with a free energy about $27 \mathrm{~kJ} / \mathrm{mol}$ lower than that for a $7 \AA$ interionic distance.

Additionally, the Marcus relation

$\Delta A^{\neq}=\frac{\left(\Delta A^{\circ}+\alpha\right)^{2}}{4 \alpha}$

which relates the activation free energy $\Delta A^{\neq}$to the reaction free energy $\Delta A^{\circ}$ and to the solvent reorganization energy $\alpha$, predicts that in the case $\left(\Delta A^{\circ}=0\right)$ the reorganization energy should be 4 times bigger than the activation free energy, which is remarkably well verified by the present calculations $(\alpha=$ $4.03 \Delta A^{\neq}$for the $5 \AA$ calculation and $\alpha=3.92 \Delta A^{\neq}$ for the $7 \AA$ calculation). It should also be noticed that this relation was more poorly satisfied in the truncated electrostatics approach at $7 \AA\left(\alpha=3.52 \Delta A^{\neq}\right.$ [40]).

\section{Conclusions}

The two PMFs presented in this article clearly show that the use of Ewald sums (or an approximation such as the one used in this work) yields substantially different results in comparison with the use of truncated electrostatic interactions. Besides the obvious difference in magnitude of the values between the two PMFs, the appearance of two minima in the truncated case is perhaps even more noticeable. The first
TE minimum, corresponding to a water bridging configuration, may have a softer analogous effect in the Ewald PMF in the region up to $4 \AA$ as the free energy curve does seem to be not as steep as it is a bit farther ahead.

The second minimum of the TE PMF simply does not appear in the Ewald PMF. It was shown in this work that significant differences occur in the solvation shells of waters. The poorer orientation of the waters in the TE case may be the cause for weaker cationwater interactions, thus providing weaker shielding by the solvent and causing the free energy to rise as this orientation loss becomes more pronounced in the 5.2$6.5 \AA$ region. This effect should also be an indication that truncation of the long range electrostatic interactions overestimates the repulsion between the hydration shells of the solutes so as to cause them to distort more significantly.

The origin of the $5.2 \AA$ minimum does seem, thus, to have an important short range structural component. It is an interesting point to notice how different long range treatments may have such a marked influence on the short range structure. It should also be notice that even though both PMFs seem to tend to the proper asymptotic behavior, as the long distance TE PMF values correspond to strong interactions between the ions' solvation shells, they may in fact be a local maximum that would decay, at even longer distances, to the correct asymptotic values as these interactions became weaker.

In relation to the electron transfer studies of the present system, it was found out that the electron transfer process in fact does occur with a lower activation energy for shorter interionic separations. The process at $5 \AA$ of separation occurs with an activation free energy about $27 \mathrm{~kJ} / \mathrm{mol}$ lower than it occurs at $7 \AA$. Comparing this value with the free energy which is needed to make the two ions approach from $7 \AA$ to $5 \AA$, which can be seen from the PMF to be of about $13 \mathrm{~kJ} / \mathrm{mol}$, one can conclude that in this case the electron transfer at very short distances is indeed favorable.

In addition it should be noted that the comparison of the present results obtained for the electron transfer at $7 \AA$, which were obtained by treating the electrostatic interactions as described previously, when compared with earlier results obtained with truncation of the long range electrostatic interactions [40] clearly 
show that the latter approach is inappropriate for the solution of this problem. In fact, the value for the activation free energy obtained with that model is substantially higher than the analogous one obtained in the present work. Also Marcus relation is verified more satisfactorily on the present calculation than in the truncated electrostatic case.

\section{Acknowledgements}

Financial support from Fundação para a Ciência e a Tecnologia (Lisbon) through project PRAXIS/PCEX/ QUI/61/96 is acknowledged. A.P.C. wishes to thank the Programa Praxis XXI for a doctoral scholarship.

\section{References}

[1] R.A. Marcus, J. Chem. Phys. 24 (1956) 966.

[2] R.A. Marcus, J. Chem. Phys. 24 (1956) 979.

[3] R.A. Marcus, J. Chem. Phys. 43 (1965) 679.

[4] M.D. Newton, N. Sutin, Ann. Rev. Phys. Chem. 35 (1987) 437.

[5] A.L. Magalhães, B. Maigret, J. Hoflack, J.A.N.F. Gomes, H.A. Scheraga, J. Protein Chem. 13 (1994) 195.

[6] J. Gao, S. Boudon, G. Wipff, J. Am, Chem. Soc. 113 (1991) 9610.

[7] S. Boudon, G. Wipff, B. Maigret, J. Phys. Chem. 94 (1990) 6056.

[8] B.M. Pettitt, P.J. Rossky, J. Chem. Phys. 84 (1986) 5835.

[9] L.X. Dang, B.M. Pettitt, J. Am. Chem. Soc. 109 (1987) 5531.

[10] J.K. Buckener, W.L. Jorgensen, J. Am. Chem. Soc. 111 (1989) 2507.

[11] W.L. Jorgensen, J.K. Buckner, S.E. Huston, P.J. Rossky, J. Am. Chem. Soc. 109 (1987) 1891.

[12] E.C. Zhong, H.L. Friedman, J. Phys. Chem. 92 (1988) 1685.

[13] H. Xu, H.L. Friedman, J. Solution Chem. 19 (1990) 1155.

[14] L.X. Dang, B.M. Pettitt, P.J. Rossky, J. Chem. Phys. 96 (1992) 4046.

[15] J.S. Bader, D. Chandler, J. Phys. Chem. 96 (1992) 6423.

[16] E. Guàrdia, R. Rey, J.A. Padró, J. Chem. Phys. 95 (1991) 2823.

[17] O.A. Karim, J. Chem. Phys. 96 (1992) 9237.
[18] G. Hummer, D.M. Soumpasis, M. Neumann, Mol. Phys. 81 (1994) 1155.

[19] G.S. Del Buono, F.E. Figueirido, R.M. Levy, Chem. Phys. Lett. 263 (1996) 521.

[20] F. Figueirido, G.S.D. Buono, R.M. Levy, J. Chem. Phys. 103 (1995) 6133.

[21] J.E. Roberts, J. Schnitker, J. Chem. Phys. 101 (1994) 5024.

[22] D.J. Adams, G.S. Dubey, J. Comput. Phys. 72 (1987) 156.

[23] H.J.C. Berendsen, J.P.M. Postma, W.F. van Gunsteren, J. Hermans, in: B. Pullman (Ed.), Intermolecular Forces, Reidel, Dordrecht, 1981.

[24] L. Curtiss, J.W. Halley, X.R. Wang, Phys. Rev. Lett. 69 (1992) 2435.

[25] M.N.D.S. Cordeiro, Ph.D. Thesis, University of Porto, Porto, 1995.

[26] M. Nomura, T. Yamaguchi, J. Phys. Chem. 92 (1988) 6157.

[27] P.S. Salmon, G.W. Neilson, J.E. Enderby, J. Phys. C 21 (1988) 1335.

[28] F.A. Cotton, G. Wilkinson, Advanced Inorganic Chemistry, 5, John Wiley and Sons, New York, 1988.

[29] M.N.D.S. Cordeiro, R. Cammi, J.A.N.F. Gomes, J. Tomasi, Theor. Chim. Acta 82 (1992) 165.

[30] M.P. Allen, D.J. Tildesley, Computer Simulation of Liquids, Oxford University Press, Oxford, 1987.

[31] F.M.S.S. Fernandes, B.J.C. Cabral, Portugal. Phys. 18 (1987) 39.

[32] F.F.M. Freitas, B.J.C. Cabral, F.M.S.S. Fernandes, J. Phys. Chem. 97 (1993) 9470.

[33] F.F.M. Freitas, B.J.C. Cabral, F.M.S.S. Fernandes, AIP Conf. Proc. 330 (1995) 309.

[34] F.F.M. Freitas, F.M.S.S. Fernandes, B.J.C. Cabral, J. Phys. Chem. 99 (1995) 5180.

[35] J.E. Roberts, J. Schnitker, J. Phys. Chem. 90 (1995) 1322.

[36] N. Metropolis, A.W. Rosenbluth, M.N. Rosenbluth, A.H. Teller, E. Teller, J. Chem. Phys. 21 (1953) 1087.

[37] R.W. Zwanzig, J. Chem. Phys. 22 (1954) 1420.

[38] W.L. Jorgensen, C. Ravimohan, J. Chem. Phys. 83 (1985) 3050.

[39] M. Migliori, G. Corongiu, E. Clementi, J. Chem. Phys. 88 (1988) 7766.

[40] A.J.P. Carvalho, M.N.D.S. Cordeiro, J.A.N.F. Gomes, J. Mol. Struct. (Theochem) 371 (1996) 185.

[41] M. Bishop, S. Frinks, J. Chem. Phys. 87 (1987) 3675.

[42] T.P. Straatsma, J.A. McCammon, J. Chem. Phys. 95 (1991) 1175.

[43] K. Watanabe, M.L. Klein, Chem. Phys. 131 (1989) 157.

[44] J.-K. Hwang, A. Warshel, J. Am. Chem. Soc. 109 (1987) 715. 